\title{
The diagnostic value of combined indexes in Acute Renal injury after Cardiopulmonary Bypass Surgery in infants with Congenital Heart Disease
}

Haifei Yu

Fujian Maternity and Child Health Hospital

Xinrui Wang

Fujian Maternity and Child Health Hospital

Qiang Chen

Fujian Union Hospital

Liangpu Xu

Fujian Maternity and Child Health Hospital

Hua Cao ( $\nabla$ caohua69@fjmu.edu.cn )

Fujian Maternity and Child Health Hospital

Research article

Keywords: Cardiopulmonary bypass, Risk factors, AKI, Non-cyanotic CHD, infants

Posted Date: March 3rd, 2020

DOl: https://doi.org/10.21203/rs.3.rs-15732/v1

License: (9) This work is licensed under a Creative Commons Attribution 4.0 International License.

Read Full License 


\section{Abstract}

Objective: To investigate the incidence and risk factors of acute renal injury (AKI) after cardiopulmonary bypass (CPB) cardiac surgery in infants with congenital heart disease(CHD).

Methods: Single-center data from a total of 613 infants with congenital heart disease who underwent cardiothoracic surgery in Fujian Union Hospital.The included patients were divided into two groups according to the occurrence of AKI: AKI group $(n=68)$ and non-AKI group $(n=92)$. We obtained clinical data from the electronic hospitalization information system and the laboratory database. All infants were tested for serum creatinine at least twice within 12 hours of admission and after operation. We determined AKI events according to creatinine criteria for improving global prognosis of renal diseases. The general and clinical data of the infants were collected, and the related risk factors were explored by univariate analysis and Logistic regression analysis.

Results: 160 patients had congruent lab and echocardiogram data foranalysis. Most of patients are male (56.26\%). Original congenital cardiac malformation is similar with our study, the most common is left-toright shunt $\mathrm{CHD}(58 \%)$, followed by right-to-left shunt $\mathrm{CHD}(18.75 \%)$. All patients showed differences in liver function, renal function, cardiac function and inflammatory indexes within 12 hours of admission and after operation $(\mathrm{p}<0.05)$. The AKI group and non-AKI group showed Significant statistical difference in arein age, serum myocardial enzyme , hepatic function, ejection fraction , hemoglobin , platelet count were significantly different meaning $(\mathrm{p}<0.05)$. Regression analyses showed that blood oxygen saturation (95\% Cl 1.003-2.999), CREA(95\% Cl 1.070-1.253), UREA(95\% Cl 1.1803.325), $\mathrm{CRP}(95 \% \mathrm{Cl} 1.006-1.058), \mathrm{BNP}(95 \% \mathrm{Cl} 0.999-1.000)$ at 12 hours postoperatively, and in admission to PCT (95\% Cl 0.461-0.936), $\mathrm{Neu}(95 \% \mathrm{Cl} 0.909-0.995), \mathrm{ALP}(95 \% \mathrm{Cl} 1.070-1.253)$, nadir intraoperative renal regional tissue oximetry to be independent predictors of postoperative kidney damage as measured by blood oxygen saturation, hepatic function, kidney function, cardiac function , Serum myocardial enzyme, inflammatory factor s and blood Routine .

Conclusions: Choosing the best age for infants's cardiac surgery, actively preventing preoperative complica- tions, postoperative pneumonia, heart failure and hypoxia play an important role in preventing AKI.

\section{Introduction}

Acute kidney injury (AKI) is a group of clinical syndromes characterized by acute renal dysfunction[1]. It was defined as abnormalities in kidney structure or function that have existed for less than 90 days[2, 3]. AKI is commonly found in high-risk hospitalized children, especially in infants,which is related to higher mortality and leads to sequelae of CKD in adults[4,5]. The incidence of AKI among children in China $(0.31 \%-1.4 \%)$ is significantly lower than that in developed countries $(17.0 \%-26.9 \%)[4,6]$. The disease burden of AKI in children is obviously underestimated [7, 8]. According to research, the Rate of AKI in community hospitals is about $2 \%$, while in large academic institutions, the rate of AKI accounts for more 
than $20 \%$ of all hospitalisations $[9,10]$. In addition, if specific hospital units are studied such as ICU, cardiac surgery,and transplant centers, the rate of AKI can be $50 \%$ or more $[11,12]$.

Today, changes inserum creatinine or urine volume, neither sensitive nor specific [13,14], are widely used to evaluate renal function and diagnose AKI. Although glomerular filtration rate (GFR) can be accurately measured in the research setting, it was a complicated technology and time consuming in practical application [15].Therefore, new AKI markers (such as NGAL and cystatin C) have been developed clinically to assess changes in renal function by monitoring solutes that normally cleared by the kidney, or by calculating the total intake or discharge amount of liquid of the patient in a specified period of time $[16,17]$.

Cardiopulmonary bypass (CPB) is an indispensable auxiliary technology for open heart surgery. Nonphysiological perfusion, embolization and inflammatory reaction during CPB can lead to AKI after operation,which will not only lead to an increase in short-term mortality, but also closely related to the long-term survival rate of patients, affecting the effect of surgery and prognosis of patients [18].

Previous studies on AKI have mostly focused on adult and adolescent patients [19-21], and the investigation and study to evaluate the acute kidney injury of infants after operation are relatively rare,complications, hospitalization expenses and other adverse consequences of AKI in hospitalized infants are no less than those of adults, and the physiological regulation function of infants's own organs is worse than that of adults, which often results in worse prognosis than adults[22, 23]. For this reason, researchers have made appropriate improvements in the diagnosis and treatment of AKI in infants with congenital heart diseases compared with adults, and tried to find more sensitive and accurate markers to monitor the occurrence and development of AKI in infants [24, 25].

Based on the diagnostic of AKIN criteria, this study retrospectively analyzed the risk factors related to AKI after CPB for infants with congenital heart disease. The purpose of this study is to find out the risk factors of AKI after neonatal cardiopulmonary bypass, and to provide clinical and theoretical basis for preventing and reducing AKI.

\section{Materials And Methods \\ Study design}

A total of 160 infants who underwent CPB cardiac surgery in the affiliated Union Hospital of Fujian Medical University from July 2018 to August 2019 were analyzed retrospectively, including 90 males and 68 females, aged 0.05 to 4 years with an average of $(1.44 \pm 1.15)$ years. The main disease type included 99 cases of left-to-rightshun CHD (ventricular septal defect, atrial septal defect), 30 cases of right-toleftshun CHD (tetralogy of fallot and transposition of great arteries), and 32 cases of non-shunt CHD (pulmonary stenosis, aortic stenosis). Those with non-CPB cardiac surgery, early postoperative death (within 24 hours) and preoperative chronic renal insufficiency requiring drug or dialysis were excluded. There was no significant difference in height, heart rate, blood oxygen saturation, white blood cell count, 
creatinine and urea between the two groups $(P>0.05)$, which was comparable. With the consent of the hospital ethics committee, the family members of the infants all signed informed consent forms before the operation.

The case Grouping Scheme(inclusion criteria and exclusion criteria) is described in Fig. 1. AKI was defined as serum creatinine (Scr) increase $\geq 26.4 \mu \mathrm{mol} / \mathrm{L}$ within $48 \mathrm{~h}$, or increase $\geq 50 \%$ from the basic value; and/or urine volume $<0.5 \mathrm{ml} /(\mathrm{kg} / \mathrm{h})$ for $6 \mathrm{~h}$ [26]. Infants were divided into 2 groups according to whether AKI occurred, 68 cases in AKI group and 92 cases in non-AKI group. The clinical data of age, sex, body mass, heart rate, blood oxygen saturation, preoperative and postoperative basic scr value, preoperative basic blood urea value (UREA), ejection fraction (EF), hemoglobin ( $\mathrm{Hb}$ ), white blood cell count, platelet count, hematocrit (HCT), congenital heart disease type, liver function index, myocardial enzyme examination, heart failure marker, inflammation index and other relevant clinical data were collected.

\section{Statistical analysis}

All data were statistically processed by SPSS 23.0 , the normal measurement data were expressed as $( \pm$ s), and the data between groups was compared by independent sample t-test: Counting data is expressed as cases/percentage, and the comparison between groups were compared with $\chi 2$ test. Median/quartile spacing of skewness data is expressed, and non-parametric rank sum test is used for inter-group comparison. One way ANOVA included significant measurement factors into the calculation, taking AKI or non-AKI as a dependent variable and carrying out binary multivariate logistic regression analysis, $p<0.05$ was defined as statistically significant.

\section{Results}

The average ages of infants in AKI group and non-AKI group were $1.63 \pm 1.28$ and $1.30 \pm 1.16$. The ratio of living Area and types-of-cardiac-shunt, UREA, BNP, HGB in non-AKI group are higher than those in AKI group, while age, height, blood oxygen saturation, PLT, WBC are lower than those in AKI group. These indexes, Weight $(P=0.038)$, SPO2 $(P=0.001)$, NT-proBNP $(P=0.028)$ and PLT $(P=0.054)$, showed statistically significant difference.The clinical characteristics are shown in Table 1.

Table 2 lists the comparison of the basic examination values of various clinical indexes at admission and the retest values within 12 hours after operation. Infants suffered from CHD and surgical operation, resulting in abnormal clinical examination indexes of kidney function, hepatic function, cardiac function, serum myocardial enzyme, inflammatory factors blood routine and other organ systems. We observed that AST, TB, CREA, UREA, NT-proBNP, LDH, CK, CKMB, WBC, CRP, PCT were significantly higher than those before operation $(p<0.05$ or $p<0.01)$. There was no statistical difference between HGB and EF, $(P=0.143$, $0.248)$.

In Table 3, the laboratory results of blood samples from AKI and non-AKI CHD infants showed that multiple organ systems' variables, such as $\operatorname{ALT}(P=0.026), \operatorname{AST}(P=0.022), \operatorname{CREA}(P=0.000), \operatorname{UREA}(P=$ 
0.000), $B N P(P=0.076), C K M B(P=0.054), C R P(P=0.010)$, changed after operation under cardiopulmonary bypass.

Univariate Analysis of infants with Acute Renal Injury showed that there was no statistical difference in height, body mass, HR,EF, ALT, AST, PLT, HGB, TB, Y-GT, LDH, CK, CKMB, WBC, preoperative NT-proBNP, proactive $P C T$, and proactive ALP between the AKI and non-AKI group $(P>0.05)$. However, Those independent variables including preoperative CREA $(95 \% \mathrm{Cl} 0.901-0.987)$ and postoperative CREA $(95 \% \mathrm{Cl}$ 1.103-1.295), preoperative UREA (95\% Cl 0.513-0.965), postoperative UREA(95\% Cl 1.586-3.748), preoperative PCT $(95 \% \mathrm{Cl} 0.484-0.902)$, postoperative CRP (95\% Cl 1.002-1.051), Neu (95\% Cl 0.9120.982), postoperative $\mathrm{BNP}(95 \% \mathrm{Cl} 0.999-1.000)$, preoperative $\mathrm{ALP}(95 \% \mathrm{Cl} 0.998-1.010)$, and blood oxygen saturation $(95 \% \mathrm{Cl} 1.252-3.696)$ showed statistical significance in table $4(\mathrm{p}<0.05)$. Multivariate logistic regression analysis indicated that age $\leq 1$ year old, blood oxygen saturation(1.003-2.999), postoperative CREA (95\%Cl1.070-1.253), UREA(95\%C1.180-3.325), CRP(95\%Cl1.006-1.058), BNP(95\%C0.999-1.000), and preoperative PCT(95\%Cl0.461-0.936), Neu(95\%Cl0.909-0.995), ALP(95\%Cl1.070-1.253),were risk factors for AKI in infants with congenital heart disease undergoing open heart surgery under cardiopulmonary bypass in table 5 .

\section{Discussion}

Researches have revealed that there are many factors related to AKI in CHD infants, including congenital hypoplasia, low physiological function, surgical trauma, receiving nephrotoxic drugs therapy, primary or secondary kidney diseases, dehydration and congenital malformation of urinary system [22, 28]. Notablely, pathogenic factors of AKI vary greatly due to different countries, geographical environment, living standards and available resources[27, 28]. Recently, a multi-center prospective study on the epidemiology of AKI in critical infants patients showed that those from pediatric care centers in Asia, Australia, Europe and North America had AKI, with an incidence rate of $26.9 \%$, among which $11.6 \%$ developed severe AKI [23]. In 2017, an epidemiological survey conducted pAKI from Belgium showed that the incidence rate of AKI hospitalized infants was $0.59 \%, 27 \%$ of which turned into chronic kidney disease, with a mortality rate as high as $15 \%$ [22]. Therefore, it is of great significance to explore the risk factors related to AKI after congenital heart disease surgery in children. In this study, we aim to promote the early screening and diagnosis of high-risk population by clinicians, and take corresponding early preventive measures to reduce the incidence of AKI after surgery, and to achieve timely diagnosis and treatment and improve the prognosis. The epidemiological characteristics of AKI in infants with congenital heart diseases are different from those in adults. CREA in infants with AKI can significantly increase in the early postoperative period. Infants without significant increase in CREA on the 1st and 2nd postoperative days are unlikely to develop AKI (negative predictive values are $87 \%$ and $98 \%$, respectively[29]. AKI is one of the common and serious complications after CPB, which has adverse effects on the prognosis of patients and even increases the mortality rate after CPB [30]. AKI is commonly found in high-risk hospitalized children, which is related to higher mortality and leads to sequelae of CKD in adults [31]. The incidence of AKI among children in China (0.31\%-1.4\%) is significantly lower than that 
in developed countries (17.0\%-26.9\%), The disease burden of AKI in children is obviously underestimated [32].

Reviewing the relevant research results on AKI risk factors after pediatric congenital heart disease surgery at home and abroad, the results are not the same, which may be due to the differences in the size of the research institutions, the age groups of the research subjects and the influencing factors included in the research. Some studies have analyzed the risk factors of AKI in children aged 0-18 years after congenital heart disease surgery, and found that the younger the children, the higher the probability of AKI occurrence [33,34], which is related to the gradually mature renal function of children after birth [35]. Therefore, this study aims at children younger than 4 years old with higher risk, and comprehensively includes the effects of various organs and systems that may affect AKI, including liver function, renal function, cardiac function, inflammatory response, blood cells, in order to obtain more scientific evaluation factors. The results of this study showed that the incidence of AKI was $42.5 \%$. After logistic regression analysis, blood oxygen saturation decreased; Increase in Value to BNP,CREA ,CRP and UREA were measured within 12 hours after operation and NEu, PCT at admission were independent risk factors for postoperative AKI in under 4-year-old children with congenital heart disease.These were similar to previous research results. Renal hypoperfusion, sepsis and toxic drugs can cause acute tubular necrosis[36, 37]. Intrarenal vasoconstriction caused by various reasons leads to endothelial cell dysfunction, imbalance of vasomotor substances and loss of local self-regulation ability. At the same time, the release of angiotensin II (Ang II) increased, the feedback of tubules and the release of inflammatory mediators led to continuous vasoconstriction and decreased glomerular filtration rate. Blood redistribution, causing damage to renal tubular cells with high oxygen demand at the junction of cortex and medulla[38, 39].

Creatinine is mainly formed by conversion of creatine phosphate in muscle through spontaneous irreversible non-enzymatic dehydration reaction, and then removed by kidney. Endogenous creatinine content is related to muscle content and quality, and the muscle content of children increases gradually after birth. In fact, the endogenous creatinine levels of different ages and individuals are different [40,41], so the renal function of children cannot be estimated simply by Scr level, and it is difficult to avoid the loss of actual measurement errors and other excretory pathways when estimating according to the change value of urine volume of children. Therefore, the design of this study is based on the multi-system detection value of infants combined with renal function, which is a more comprehensive plan that is more closely related to the actual clinical condition. Hypoxia inducible factor (HIF) is a transcription factor, HIF binds to hypoxia response elements of target genes to play a specific role. In ischemic diseases such as kidney diseases, cerebrovascular diseases and myocardial ischemia, HIF often plays a protective role under the attack of acute hypoxia. However, many studies have found that HIF has the harmful side of fibrosis and apoptosis, which may be related to chronic hypoxia environment [42-44].

There are some limitations to this study. First of all, the research center is not selected by random sampling and may be affected by selection bias. Second, our research subjects come from many areas in China's construction provinces, including most coastal areas and some non-coastal areas, and all the 
children in the group are congenital heart disease children, without other disease groups and normal control groups, resulting in the lack of more credible evidence for the results. Third, we cannot use urine volume to identify AKI because we do not have these data in our cohort, which may lead to underestimation of the incidence of AKI. In addition, There are some similarities with the views of the literature, but there are also some differences. The difference may be due to the relatively small sample size of this study and the research The research factors are not comprehensive, which has a certain impact on the research results.

\section{Conclusion}

To sum up, many adverse outcome events can be induced after open surgery under cardiopulmonary bypass for infants with congenital heart disease. Choosing the best age for infant surgery, actively preventing preoperative complications, preventing infection and heart failure are important measures to prevent AKI. Early detection and treatment can be realized by avoiding or reducing the screened risk factors, so as to reduce the incidence of postoperative AKI and ensure the health of infants to the greatest extent.

\section{Abbreviations}

AKI: acute kidney injury; CHD: congenital heart disease; NGAL:Neutrophil gelatinase-associated lipocalin, apolipoprotein-2; CPB: cardiopulmonary bypass; $\mathrm{PCHD}$ : premature coronary heart disease; ALT: alanine transaminase; TB: total bilirubin; AST: alanine transaminase; ALP: alkaline phosphatase; EF: ejection fraction; HR: heart rate; CREA: Serum creatinine; UREA: Serum urea; NT-proBNP: NT-brain natriuretic peptide; LDH: Lactic dehydrogenase; CK: Creatine kinase; CKMB: Creatinekinase-MB/CK2; WBC: leukocyte count; NEU: neutrophil count; $\gamma$-GT: $\gamma$-glutamyl transpeptidase; CRP: C-reactive protein; PCT: procalitonin; HGB:hemoglobin; PLT: platelet count; SPO2: blood oxygen saturation.

\section{Declarations}

\section{Acknowledgements}

We highly acknowledge the contribution by the participating doctors: Xiaofu Dai, Dongshan Liao, Xueshan Huang.

\section{Authors' contributions}

Haifei Yu performed the data analysis and drafted the manuscript. Xinrui Wang, Qiang Chen, and Liangpu Xu participated in this study. Hua Cao designed the study and revised the drafted article. All authors read and approved the final manuscript.

\section{Funding}


This work was sponsored by the Young and Middle-Aged Talent Training Project of Fujian Province of China (grand No. 2014-ZQN-ZD-12) (Hua Cao) and the Fujian Maternity and Child Health Hospital of Fujian Province of China (grant No.YCXB 18-05 and No.YCXM 19-03) (Xinrui Wang).

\section{Availability of data and materials}

Data sharing not applicable to this article as no data sets were generated or analyzed during the current study.

\section{Ethics approval and consent to participate}

This study was approved by the ethics committee of the Fujian Medical University, China. All participants were informed of the study in detail and signed a consent form.

\section{Consent for publication}

Not applicable.

\section{Competing interests}

The authors declare that they have no competing interests.

\section{References}

1. Li PK, Burdmann EA, Mehta RL. Acute kidney injury: Global health alert. Transplantation. 2013; 95( 5): 653-657.

2. Sparrow HG, Swan JT, Moore LW, et al. Disparate outcomes observed within Kidney Disease: Improving Global Outcomes (KDIGO) acute kidney injury stage 1. Kidney Int. 2019; 04. 95(4): 905913.

3. Chawla LS, Bellomo R, Bihorac A, et al. Acute kidney disease and renal recovery: consensus report of the Acute Disease Quality Initiative (ADQI) 16 Workgroup. Nat Rev Nephrol 2017; 13: 241-57.

4. Kaddourah A, Basu RK, Bagshaw SM, Goldstein SL; AWARE Investigators: Epidemiology of acute kidney injury in critically ill children and young adults. N Engl J Med. 2017;376: 11-20.

5. Bellomo R, Kellum JA, Ronco C: Acute kidney injury. Lancet. 2012; 380: 756-766.

6. GM, Shirey-RiceJ,Chen L, ByrneDW,VanDriest SL: Acute kidney injury incidence in noncritically ill hospitalized children, adolescents, and young adults: A retrospective observational study. Am J Kidney Dis. 2016;67: 384-390.

7. Waikar SS, Wald R, Chertow GM, Curhan GC, WinkelmayerWC, Liangos O, SosaMA, Jaber BL:Validity ofinternational classifification of diseases, ninth revision, clinical modifification codes for acute renal failure. J Am Soc Nephrol. 2006;17: 1688-1694.

8. Lafrance JP, Miller DR: Defifining acute kidney injury in database studies: The effects of varying the baseline kidney function assessment period and considering CKD status. Am J Kidney Dis. 2010;56: 
651-660.

9. Ronco C. Epidemiology of acute kidney injury in critical care. In: Nephrology CC, ed.Elsevier. Philadelphia, PA.2019: 65-75.

10. Malhotra R, Bouchard J, Mehta RL. Community- and hospital-acquired acute kidney injury. In: Critical Care Nephrology (third edn) Elsevier: Philadelphia, PA. 2019: 75-80.

11. Hoste EA, Bagshaw SM, Bellomo R, et al. Epidemiology of acute kidney injury in critically ill patients: the multinational AKI-EPI study. Intensive Care Med. 2015; 41: 1411-23.

12. Srisawat N, Kulvichit W, Mahamitra N, et al. The epidemiology and characteristics of acute kidney injury in the Southeast Asia intensive care unit: a prospective multicentre study. Nephrol Dial Transplant. 2019; published online May 10. DOI:10.1093/ndt/gfz087.

13. Delanaye P, Cavalier E, Pottel H. Serum creatinine: not so simple! Nephron 2017; 136: 302-08.

14. Ronco C, Bellomo R, Kellum J. Understanding renal functional reserve. Intensive Care Med 2017; 43: 917-20.

15. Stevens LA, Levey AS. Measured GFR as a confirmatory test for estimated GFR. J Am Soc Nephrol 2009; 20: 2305-2313.

16. Stevens LA, Coresh J, Schmid CH, Feldman HI. Estimating GFR using CystatinC alone or in combination with creatinine: a pooled analysis of 3418 individuals with CKD. Am J Kidney Dis. 2008;51: 395-406.

17. Hirsch R, Dent C, Pfriem $\mathrm{H}$, et al. NGAL is an early predictive biomarker of contrast-induced nephropathy in children. Pediatr Nephrol. 2007; 22(12):2089-2095.

18. Kumar AB,Suneja M. Cardiopulmonary bypass-associated acute kidney injury. Anesthesiology. 2011;114(4):964-6.

19. Chanchlani R, Nash DM,et al. Secular Trends in Incidence, Modality and Mortality with Dialysis Receiving Aklin Children in Ontario: A Population-Based Cohort Study. Clin J Am Soc Nephrol. 2019.Sep 06;14(9):1288-1296.

20. Greenberg JH, Zappitelli M, Jia Y, et al. Biomarkers of AKI Progression after Pediatric Cardiac Surgery.J. Am. Soc. Nephrol. 2018.05;29(5):1549-1556.

21. Xin Xu, Sheng Nie, Aihua Zhang, Jianhua Mao, et al. Acute Kidney Injury among Hospitalized Children in China. Clin J Am Soc Nephrol. 2018;13: 1791-1800.

22. Keenswijk W, Vanmassenhove J, Raes A, et al. Epidemiology and outcome of acute kidney injury in children, a single center study. Acta Clinica Belgica; 2017:1-8.

23. Nakhjava-shahraki B, Yousefifard M, Ataein, et al. Accuracy of cystatin C in prediction of acute kidney injury in children; serum or urine levels:which one works better? A systematic review and metanalysis. BMC Nephrol ,2017;18(1):120.

24. Ozcakar Z B, Yalcinkaya F, Altas B, et al. Application of the new classification criteria of the Acute Kidney Injury Network: a pilot study in a pediatric populatio. Pediatr Nephrol. 2009; 24(7): 1379-1384. 25. Claudio Ronco, Rinaldo Bellomo, John A Kellum. Acute kidney injury. Lancet 2019; 394: $1949-64$. 
26. Basu RK, Wang Y, Wong HR, et al. Incorporation of biomarkers with the renal angina index for prediction of severe AKI in critically ill children. Clin J Am Soc Nephrol. 2014; 9(4): 654-662.

27. Hirsch R, Dent $C$, Pfriemc $H$, et al. NGAL is an early predictive biomarker of contrastinduced nephropathy in children. Pediatr Nephrol. 2007; 22(12): 2089-2095.

28. Claudio Ronco, Rinaldo Bellomo, John A Kellum. Acute kidney injury. Lancet 2019; 394:1949-64.

29. Basu RK, Wang Y, Wong HR, et al. Incorporation of biomarkers with the renal angina index for prediction of severe AKI in critically ill children. Clin J Am Soc Nephrol. 2014; 9(4):654 662.

30. Kaddourah A, Basu RK, Bagshaw SM, et al. Epidemiology of acute kidney injury in Critically ill children and young adults. The New England journal of medicine, 2017;376 (1): 11-20.

31. Zappitelli M, Bernier P L, Saczkowski R S, et al. A small post-operative rise in serum Creatinine predicts acute kidney injury in children undergoing cardiac surgery. Kidney Int. 2009;76(8): 885-892.

32. Mao H囚Katz N, Ariyanon W, et al. Cardiac surgery- associated acute kidney injury. Blood Purif, 2014, 37 (Suppl 2): 34-50.

33. McGregor TL, Jones DP, Wang L, Danciu I, Bridges BC, Fleming GM, Shirey-RiceJ,Chen L, ByrneDW,VanDriest SL: Acute kidney injury incidence in noncritically ill hospitalized children, adolescents, and young adults: A retrospective observational study. Am J Kidney Dis. 2016;67: 384390.

34. Li S, Krawczeski C D, Zappitelli M, et al. Incidence, risk factors, and outcomes of acute kidney injury after pediatric cardiac surgery: a prospective multicenter study. Crit Care Med.2011;39(6):1493-1499.

35. Watkins S C, Williamson K, Davidson M, et al. Long-term mortality associated with acute kidney injury in children following congenital cardiac surgery. Paediatr Anaesth. 2014;24( 9):919 -926.

36. Botwinski C A, Falco G A. Transition to postnatal renal function. J Perinat Neonatal Nurs. 2014;28(2):150 -154区

37. Lameire NH, Bagga A, Cruz D, et al. Acute kidney injury: an increasing global Concern. Lancet, 2013, 382 (9887): 170-179.

38. Bonventre JV, Yang L. Cellular pathophysiology of ischemic acute kidney injury. J Clin Inve 2011; 121 (11) : 4210-4221.

39. Bellomo R, Kellum JA, \onco C. Acute kidney injury. Lancet. 2012; 380( 9857): 756-66.

40. Baxmann A C, Ahmed M S, Marques N C, et al. Influence of muscle mass and physical activity on serum and urinary creatinine and serum cystatin C. Clin J Am Soc Nephrol. 2008;3(2): 348-354.

41. Inaba $M$, Kurajoh $M$, Okuno $S$, et al. Poor muscle quality rather than reduced lean body mass is responsible for the lower serum creatinine level in hemodialysis patients with diabetes mellitu. Clin Nephrol. 2010; 74(4) : 266-272.

42. Nangaku M, Rosenberger $C$, Heyman $S N$, et al. Regulation of hypoxia-inducible factor in kidney disease. Clin Exp Pharmacol Physiol. 2013;40( 2): 148 -157.

43. Satwat $H$, Suhail AS. Hypoxia-inducible factor-1 alpha in the heart:a double agent? Cardiol Rev. 2012; 20(6) : 268-273. 
44. Neetu S, Gaurav S, Vikas M, et al. Hypoxia inducible factor-1: Its potential role in cerebral Ischemia. Cell Mol Neurobiol. 2012; 32( 4):491-507.

\section{Tables}

Due to technical limitations, the tables are only available as a download in the supplemental files section.

\section{Figures}

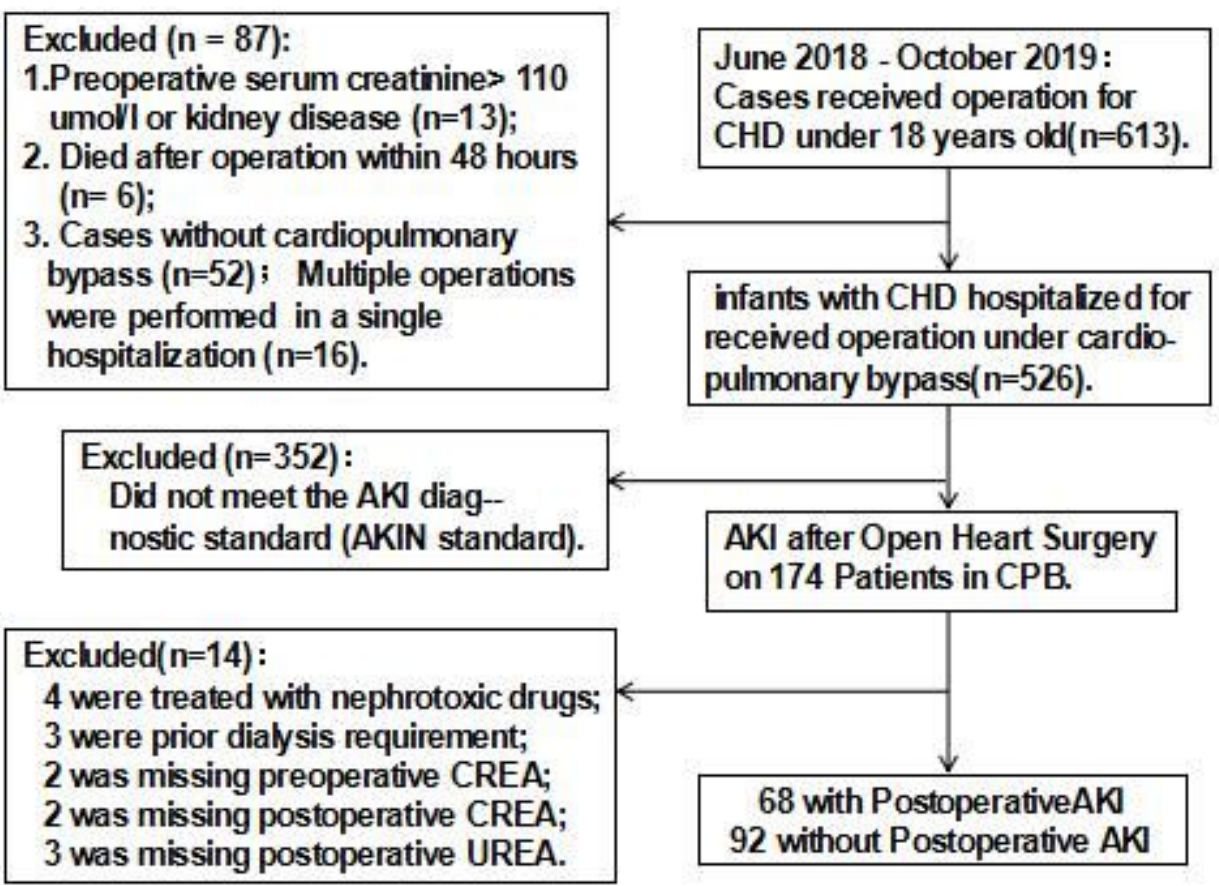

Figure 1

Study population. A total of 613 infants underwent open cardiac surgery during the study period. 160 CHD infants met the inclusion criteria.

\section{Supplementary Files}

This is a list of supplementary files associated with this preprint. Click to download.

- Table1.xlsx

- Table3.xlsx

- Table4.xlsx

- Table5.xlsx

- Table2.xlsx 